\title{
Diversidade, mecanismos de atuação e potencial agrícola de bactérias promotoras de crescimento de plantas, usando milho como cultura exemplo
}

\author{
Diversity, acting mechanisms and agricultural potential of \\ plant growth promoting bacteria, using corn as an example
}

'Universidade Federal Rural de Pernambuco (UFRPE), Rua Dom Manoel de Medeiros, s/n, Dois Irmãos, CEP 52171-900, Recife, PE, Brasil 2IPA, Recife, Pernambuco, Brasil.

*autor correspondente \mario.alirajr@ufrpe.br
Johny Jesus Mendonça' ${ }^{1}$, Mario Andrade Lira Junior ${ }^{*}$, Eric Xavier Carvalho ${ }^{2}$

Giselle Gomes Monteiro Fracetto ${ }^{1}$, Felipe José Cury Fracetto ${ }^{1}$, Michelle Justino Gomes Alves ${ }^{1}$, José de Paula Oliveira²
RESUMO: As Bactérias Promotoras de Crescimento de Plantas (BPCP) começaram a ser mais intensivamente estudadas nos últimos anos, em função do crescente interesse no aumento da sustentabilidade agrícola. Devido a esse interesse, o número de publicações no tema tem crescido exponencialmente, o mesmo ocorrendo com a diversidade biológica que é encontrada para estas bactérias em muitas espécies vegetais e ambientes. Além da diversidade biológica, também há grande variedade de meios de atuação propostos para esta promoção de crescimento, com tendência de maior efeito nas plantas cultivadas sob estresses abióticos, particularmente seca. O potencial de uso destas bactérias sob condições de campo pode ser exemplificado pelo crescente uso na cultura do milho, que é a segunda mais importante para o mercado de inoculantes agrícolas no Brasil.

PALAVRAS-CHAVE: Endofíticas, inoculante, Zea mays, gramíneas.
ABSTRACT: Plant growth-promoting bacteria $(P G P B)$ started to be more intensively studied in the last years due to increasing interest in agricultural sustainability. Due to this interest, publication in this theme has increased exponentially, which also occurs with biological diversity found for these bacteria in several plant species and environments. Besides biological diversity, there is also great variation in how these bacteria act to promote plant growth, tending to present greater effects in plants grown under abiotic stresses, particularly drought. These bacteria potential which is already the second most important crop for the Brazilian agricultural inoculant market.

KEYWORDS: Endophytic, inoculant, Zea mays, grass. may be exemplified by the increasing use of corn,

\section{Introdução}

Em meados do século XX, o interesse pelos benefícios das bactérias às plantas já se despertava pelos pesquisadores ocidentais, com estudos da então União Soviética sobre bactérias benéficas em raízes de plantas. No entanto, o difícil acesso aos trabalhos soviéticos, associado à falta de análises estatísticas, atrapalhava a aceitação desses estudos, levando a algum descrédito por parte dos ocidentais (BURR; CAESAR, 1984).

Somente em 1978, dados resultantes de experimentação realizada por Kloepper (1978) passaram a ser aceitos pela comunidade científica internacional. Esse trabalho propôs, pela primeira vez, o termo "plant growth-promoting rhizobacteria (PGPR)", derivando, em seguida, a denominação bactérias 
promotoras do crescimento de plantas (BPCPs ou PGPB, na sigla em inglês).

As BPCPs consistem em um amplo grupo de micro-organismos, habitando qualquer parte da planta (filosfera e tecidos internos) e rizosfera sem provocar prejuízos ao seu hospedeiro, desenvolvendo algum mecanismo direto e/ou indireto que promova melhorias no crescimento e desenvolvimento das plantas (SARKAR; REINHOLD-HUREK, 2014; PIETERSE; DE JONGE; BERENDSEN, 2016; BASU; RABARA; NEGI, 2017). Nos últimos anos, o aumento no interesse de uma agricultura sustentável e ecológica levou os produtores a reduzir o uso de fertilizantes químicos e ao aumento do uso de compostos naturais e práticas como a inoculação das culturas com BPCPs (GARCIA et al., 2017), já que o uso indiscriminado de fertilizantes pode provocar problemas ambientais, como a eutrofização de águas superficiais e subterrâneas, poluição do solo e emissão de gases de efeito estufa (MARKS et al., 2013; PAREDES et al., 2020; XIA et al., 2020).

Assim, não surpreende o crescente interesse pelas BPCPs no final do século XX até os dias atuais, traduzido em grande número de trabalhos publicados. Em 1990, houve apenas a publicação de quatro trabalhos. Um número ínfimo quando comparado às 503 publicações ocorrentes em 2019 e aos 204 trabalhos já publicados até meados de abril de $2020 \mathrm{em}$ levantamento na base Scopus.

As bactérias endofíticas têm sido isoladas e caracterizadas de diversos gêneros da família Poaceae e utilizadas como inoculantes (CARDOSO et al., 2010; SILVA et al., 2012; HUNGRIA; NOGUEIRA; ARAUJO, 2016; PATEL; ARCHANA, 2017), com grande potencial no aumento da produtividade e redução de custos, diminuindo a dependência de fertilizantes industrializados (CARVALHO et al., 2017).

\section{Mecanismos de Promoção de Crescimento}

As BPCPs promovem crescimento das plantas por diferentes mecanismos que podem ser classificados como biofertilizantes, fitoestimuladores e biopesticidas, com certas bactérias tendo aplicações sobrepostas, sendo que a maioria atua por mais de um mecanismo (BHATTACHARYYA; JHA, 2012), o que é desejável para produção de inoculantes (FERNANDES-JÚNIOR et al., 2015), embora seja difícil determinar as contribuições relativas de diferentes processos responsáveis pela promoção do crescimento das plantas.

Entre os mecanismos de promoção de crescimento vegetal por bactérias podem ser incluídos: facilita a absorção de nutrientes pelas plantas (SOUSA et al., 2016; GRANADA et al., 2018; ROTARU; RISNOVEANU, 2019; OLIVEIRA-LONGATTI et al., 2020); altera a disponibilidade e a toxicidade de metais às plantas (WOOD et al., 2016); protege as plantas pela indução na produção de fito-hormônios, como os ácidos salicílico e jasmônico (LIAN et al., 2000; MCCUE et al., 2000; GUTJAHR; PASZKOWSKI, 2009; DAR et al., 2015); aumenta o sistema radicular com a produção de ácido indolacético (PUENTE et al., 2019; SHEN et al., 2019; VALDEZ-NUÑEZ et al., 2019; PURI; PADDA; CHANWAY, 2020); auxilia nos processos como a fixação de nitrogênio atmosférico (FUKAMI; CEREZINI; HUNGRIA, 2018; IKEDA et al., 2020; MAHMUD et al., 2020; MORETTI et al., 2020); atua na produção de sideróforos (ZAREI et al., 2019; KOUR et al., 2020; PURI; PADDA; CHANWAY, 2020; TORRES et al., 2020); redução do efeito de estresse oxidativo (FUKAMI et al., 2018; SARKAR et al., 2018; HARMAN; UPHOFF, 2019; JOCHUM et al., 2019; YOOLONG et al., 2019); e como controle biológico e redutor do efeito de fitopatógenos (STURZ; NOWAK, 2000; TIAN; YANG; ZHANG, 2007; VERMA; WHITE, 2018).

A solubilização de formas menos solúveis deste nutriente pode assumir papel importante na nutrição vegetal (RAMAKRISHNA; YADAV; LI, 2019), já que parte do $\mathrm{P}$ do solo encontra-se imobilizado nas formas inorgânicas com cálcio, ferro e alumínio, ou em formas orgânicas (LI et al., 2017a). Estes micro-organismos são particularmente importantes para plantas dependentes da fixação biológica de nitrogênio, favorecendo a promoção do crescimento da planta em ambientes com baixa disponibilidade de P (ARAÚJO; SAMPAIO; SCHAEFER, 2017).

As bactérias mobilizam fosfatos inorgânicos insolúveis da matriz mineral do solo, podendo ser absorvidos pelas raízes das plantas (LYU et al., 2016), através da liberação de ácidos orgânicos, de baixo peso molecular, que através dos seus grupos hidróxilo e carbóxilo, quelam cátions ligados ao fosfato, convertendo-os, assim, em formas solúveis (ARCHANA; BUCH; KUMAR, 2012). A liberação destes compostos também promove a acidificação do ambiente do solo circundante, resultando na liberação de fósforo de complexos de cálcio de baixa solubilidade (MENDES et al., 2014; LI et al., 2015).

Diversos autores obtiveram isolados com potencial in vitro para solubilização de fosfato de espécies vegetais como Dodonaea viscosa L. (AFZAL et al., 2017), Agrostis capillaris L. (GROBELAK; NAPORA; KACPRZAK, 2015), palma forrageira Opuntia spp e Nopalea spp (SILVA et al., 2014), milho (MARAG; SUMAN, 2018) e cana-de-açúcar (SOUSA et al., 2016), bem como resultados positivos em algumas espécies quando inoculadas com estirpes promissoras, como no milho (KUAN et al., 2016; LI et al., 2017b)

A fixação biológica de nitrogênio (FBN) é a conversão do $\mathrm{N}_{2}$ atmosférico em amônio $\left(\mathrm{NH}_{4}^{+}\right)$, realizada por diversos micro-organismos simbióticos e de vida livre, conhecidos como diazotróficos e responsáveis por contribuir na entrada de nitrogênio nos ecossistemas naturais (CUSACK; SILVER; MCDOWELL, 2009; REED; CLEVELAND; TOWNSEND, 2011; PATEL; ARCHANA, 2017). A FBN é catalisada pela enzima nitrogenase, que requer ferro e molibdênio como cofatores codificados pelos genes nif (H,D,K), (BOLHUIS et al., 2010; GONZALEZ et al., 2012), através de uma reação altamente endergônica, com elevada energia de ativação na forma de ATP e que é inibida na presença de oxigênio (CANFIELD; GLAZER; FALKOWSKI, 2010).

Estas enzimas são encontradas em muitas bactérias associativas ou endofíticas, incluindo diversos representantes dos gêneros Azospirillum, Herbaspirillum, Bacillus, Gluconacetobacter e Burkholderia (LOWMAN et al., 2016; SILVEIRA et al., 2016; TAULÉ et al., 2016; XING et al., 2016). 
O potencial para FBN, isoladamente ou associado a outros mecanismos, foi identificado e quantificado em diferentes espécies vegetais como Dodoneae viscosa L. (AFZAL et al., 2017), milho (CARVALHO et al., 2017; GARCIA et al., 2017; MARAG; SUMAN, 2018; IKEDA et al., 2020), arroz (CHAUDHARY et al., 2012; BANIK; MUKHOPADHAYA; DANGAR, 2016; BEUTLER et al., 2016; MAHMUD et al., 2020) e cana-de-açúcar (TAULÉ et al., 2012; RODRIGUES et al., 2018; MAHMUD et al., 2020).

As auxinas desempenham um grande papel no crescimento das plantas, principalmente, atuando no sistema radicular e nas respostas a estímulos ambientais (AMIN; LATIF, 2017). Por isso é amplamente estudada e tem os mecanismos de função e regulação bem demonstrados. A principal forma de auxina natural, ácido indol-3-acético (AIA), tem sua produção por isolados microbianos e é mais eficiente no crescimento das plantas do que solução pura de AIA (PUENTE et al., 2018).

O AIA pode atuar de diferentes formas, como estimular a proliferação de células vegetais e/ou o alongamento das células vegetais, ou pode induzir a transcrição da enzima vegetal ACC (1-aminocyclopropane-1-carboxylic acid) sintase, que catalisa a formação de ACC e é produzido por diferentes gêneros como Azospirillum, Bacillus, Paenibacillus, Pseudomonas, entre outros, com diferentes caminhos biossintéticos, e com produção variável de acordo com espécies (AMIN; LATIF, 2017).

As bactérias produtoras de AIA podem aumentar o crescimento da planta, especialmente a iniciação e alongamento da raiz (SHAO et al., 2015), resultando em maior massa radicular e, consequentemente, uma maior absorção de nutrientes pela planta (HUSSAIN et al., 2015; ARSHAD et al., 2016), inclusive N (SILVEIRA et al., 2016).

Diversos autores obtiveram isolados com potencial in vitro de produção de AIA, sendo eles provenientes de diferentes espécies vegetais como Dodoneae viscosa (AFZAL et al., 2019), Agrostis capillaris L. (GROBELAK; NAPORA; KACPRZAK, 2015), Tripogon spicatus (FERNANDES-JÚNIOR et al., 2015), tomate (KHAN et al., 2016), capim-pangolão (MENDONÇA et al., 2019), milho (BATISTA et al., 2018; MARAG; SUMAN, 2018; JOCHUM et al., 2019; IKEDA et al., 2020), trigo (SALA et al., 2008; CHERIF-SILINI et al., 2016; EMAMI et al., 2019) e cana-de-açúcar (SILVA et al., 2012). A longa lista de exemplos indica como esse relacionamento entre plantas e bactérias endofíticas deve ser comum na natureza.

Além disso, resultados positivos foram obtidos com a utilização de isolados produtores de AIA nas culturas de milho (PEREZ-MONTANO et al., 2014; KUAN et al., 2016; BATISTA et al., 2018; IKEDA et al., 2020), arroz (HAHN et al., 2016; THEISEN et al., 2017; SHEN et al., 2019), trigo (BARNAWAL et al., 2017; PATEL; ARCHANA, 2017; EMAMI et al., 2019), Oliveira (SILVA et al., 2017; BIZOS et al., 2020) e soja (CASSÁN et al., 2009; THEISEN et al., 2017; PUENTE et al., 2018; ARMENDARIZ et al., 2019; IGIEHON; BABALOLA; AREMU, 2019; PUENTE et al., 2019). Novamente, este largo espectro indica a grande importância potencial destes micro-organismos na promoção de uma agricultura mais sustentável.
Sideróforos são moléculas secretadas por micro-organismos que atuam na solubilização deste ferro no solo, pela sua alta afinidade a este nutriente. Estas moléculas se ligam a receptores específicos e são ativamente transportadas através da membrana celular de bactérias (SANTOS et al., 2018), e são frequentemente liberadas por BPCPs (JI; GURURANI; CHUN, 2014; SULOCHANA et al., 2014). Além dos efeitos na nutrição, com a maior disponibilidade deste nutriente, os sideróforos também podem reduzir a população de fitopatógenos, pela competição pelo ferro (NIEHUS et al., 2017).

Embora a lista de resultados na promoção de crescimento seja menor do que para outros fatores já mencionados, há efeitos promissores em espécies como Dodoneae viscosa L. (AFZAL et al., 2017), Agrostis capillaris L. (GROBELAK; NAPORA; KACPRZAK, 2015) e milho (MARAG; SUMAN, 2018).

A enzima 1-aminocyclopropane-1-carboxylic acid (ACC) deaminase atua pela degradação do ACC, que é um precursor do etileno em todos os vegetais superiores, e pode reduzir o efeito inibitório do etileno do crescimento da planta após estresses abióticos e bióticos. Assim, as plantas podem apresentar raízes e brotações mais longas e ser mais resistentes à inibição do crescimento por uma variedade de estresses indutores de etileno, quando inoculadas com bactérias produtoras da ACC deaminase (GLICK et al., 1999; BHATTACHARYYA; JHA, 2012; GROBELAK; NAPORA; KACPRZAK, 2015).

Bactérias endofíticas produtoras desta enzima já foram identificadas em diversas espécies, frequentemente sob condições de estresses ambientais como salinidade, seca ou presença de metais pesados (SHAHAROONA; ARSHAD; ZAHIR, 2006; SHAHAROONA, 2007; ARZANESH et al., 2011; AAMIR et al., 2013; PEREZ-MONTANO et al., 2014; GROBELAK; NAPORA; KACPRZAK, 2015; MARAG; SUMAN, 2018; ZHANG et al., 2018; SAGHAFI et al., 2019).

Alguns autores também têm obtido resultados em algumas espécies inoculadas com estirpes promissoras quanto à produção da ACC deaminase in vitro em diferentes culturas como tomate (KHAN et al., 2016; WIN et al., 2018), milho (ZAREI et al., 2019), trigo (BARNAWAL et al., 2017; ZHANG et al., 2018; CHANDRA et al., 2019; CHERIF-SILINI et al., 2019) e arroz (SARKAR et al., 2018), com benefícios na promoção de crescimento e rendimento sob estresse salino. Além dos efeitos na redução dos danos causados pelo estresse abiótico, a produção de ACC-deaminase pode aumentar a colonização por fungos micorrízicos e a nodulação na ervilha (BARNAWAL et al., 2014).

Diversas bactérias da rizosfera já foram identificadas com efeitos antagônicos a fungos e nematoides (STURZ; NOWAK, 2000; TIAN; YANG; ZHANG, 2007; MACHADO et al., 2012), produzindo antibióticos, toxinas e enzimas, interferindo no processo de reconhecimento planta-hospedeiro, induzindo resistência ou proporcionando o desenvolvimento da planta (SIDDIQUI; SHAUKAT, 2002; SIDDIQUI; BAGHEL; AKHTAR, 2007; STIRLING, 2017). Algumas das espécies mais frequentemente citadas são de Bacillus, e pelo menos parte destas forma endósporos e apresenta uma multiplicidade de mecanismos antagônicos (DOMENECH et al., 2006; GROVER; NAIN; SAXENA, 2009; KAKAR et al., 2018; ALOO; 
MAKUMBA; MBEGA, 2019; HASHEM; TABASSUM; FATHI ABD ALLAH, 2019; SAXENA et al., 2019; MENÉNDEZ; PAÇO, 2020; TORRES et al., 2020).

\section{Diversidade de Bactérias Endofíticas}

Durante muitos anos, a análise da diversidade de micro-organismos endofíticos se baseava na identificação daqueles que poderiam ser recuperados em meios de cultivo. No entanto, métodos dependentes de cultivo conferem vantagem seletiva a algumas bactérias e não permitem uma visão completa da população endofítica (KAUL; SHARMA; DHAR, 2016). Recentemente, o uso de abordagens moleculares (incluindo sequenciamento de nova geração) permitiu a descrição de uma maior diversidade de endófitos vegetais e tem demonstrado que sua composição é altamente subestimada.

Hardoim et al. (2015) construíram e analisaram um banco de dados de todas as sequências de 16S rRNA atribuídas a endófitos, incluindo bactérias cultivadas e não cultivadas, e descobriram que, embora as sequências pertençam a 23 filos bacterianos diferentes, quatro (Proteobacteria, Actinobacteria, Firmicutes e Bacteroidetes) abrangem 96\% do número total de sequências procarióticas endofíticas, sendo que Proteobacteria inclui mais de $50 \%$ das sequências no banco de dados. Neste filo, os isolados da subclasse Gammaproteobacteria são os mais comumente encontrados como endófitos, incluindo gêneros como Pseudomonas, Enterobacter, Pantoea, Stenotrophomonas, Acinetobacter e Serratia.

Por outro lado, os gêneros Streptomyces, Microbacterium, Mycobacterium, Arthrobacter (Actinobacteria), bem como Bacillus, Paenibacillus e Staphylococcus (Firmicutes) também estão bem representados entre os micro-organismos endofíticos. Enquanto os gêneros Azospirillum, Bacillus, Burkholderia, Herbaspirillum e Glucanoacetobacter são mais comumente usados na experimentação em grandes culturas no nível de campo, como no milho, trigo, braquiárias, arroz cana-de-açúcar (LONGHINI et al., 2016; LIN et al., 2018; LEITE et al., 2019; PEREIRA et al., 2019; SILVEIRA et al., 2019; SOUZA et al., 2019; ZAHEER et al., 2019; NARAYANASAMY; THANGAPPAN; UTHANDI, 2020), e particularmente Azospirillum como coinoculante para feijão e soja (BRASIL, 2011; CHIBEBA et al., 2020; RONDINA et al., 2020). Como esses gêneros são comuns em solos, tem sido sugerido que a comunidade microbiana endofítica constitui uma subpopulação das bactérias rizosféricas (MARQUEZ-SANTACRUZ et al., 2010; SANTOYO et al., 2016). No entanto, como as plantas conseguem selecionar um determinado grupo de endófitos, ainda não está totalmente esclarecido (KHATABI et al., 2019; KUŹNIAR; WŁODARCZYK; WOLIŃSKA, 2019; WHITE et al., 2019).

Diferentes órgãos das plantas estão associados com diferentes comunidades bacterianas em termos de diversidade e composição. $\mathrm{O}$ microbioma na endosfera da raiz é significativamente menos diverso em relação ao da rizosfera e do solo adjacente (LIU et al., 2017). Isso sugere que as raízes são filtros efetivos de habitat, restringindo a participação da comunidade em linhagens progressivamente mais restritas, à medida que os ambientes se desviam do solo para as raízes (BULGARELLI et al., 2013).
As comunidades bacterianas endofíticas das raízes são geralmente dominadas por Proteobacteria $(\sim 50 \%$ em abundância relativa), Actinobacteria ( 10\%), Firmicutes ( 10\%) e Bacteroidetes ( 10\%), Outros filos bacterianos, incluindo, mas não limitado a Chloroflexi, Cyanobacteria, Armatimonadetes, Verrucomicrobia, Planctomycetes e Nitrospirae, são comuns na endosfera radicular, mas representam uma menor fração da comunidade (SESSITSCH et al., 2012; EDWARDS et al., 2015).

Para o desenvolvimento de biofertilizantes na agricultura, é necessário entender as diferenças entre os microbiomas da raiz da planta e da endosfera da folha/parte aérea. Existem evidências de que os endófitos bacterianos das raízes das plantas são recrutados principalmente a partir do solo, que depois ascendem às hastes e folhas via apoplastos nos vasos do xilema (CHI et al., 2005). Sendo assim, os microbiomas da endosfera folha/parte aérea da planta exibem sobreposições significativas com os das raízes nos níveis taxonômico e funcional.

Tem sido demonstrado que a endosfera da raiz pode ser dominada por alguns grupos bacterianos, evidenciando uma seleção ativa de bactérias do solo para as plantas. Exemplo disso é a predominância dos gêneros Enterobacter, Pseudomonas e Stenotrophomonas, constituindo as principais unidades taxonômicas operacionais (OTUs) na endosfera radicular de arroz (Gammaproteobacteria, 30-98\%) (SESSITSCH et al., 2012; REN et al., 2015). Em alguns casos, apenas uma ou duas OTUs bacterianas dominam a endosfera dos tecidos vegetais, como observado em caules de cana-de-açúcar em que duas OTUs eram afiliadas a Pseudomonas (52\%) e Enterobacter (35,5\%) (MAGNANI et al., 2013). De forma geral, os estudos demonstram que o microbioma na endosfera da planta é muito mais simples do que no solo adjacente e que abriga assembleias distintas, em vez de subconjuntos aleatórios do microbioma do solo.

\section{Milho como Exemplo do Potencial do Uso de BPCPs na Produção Agrícola}

O milho (Zea mays (L.) corresponde a $\sim 40 \%$ da produção nacional de grãos e é mundialmente uma cultura básica para o consumo humano e forragem animal, que depende do alto consumo de fertilizantes minerais (RANUM; PEÑA-ROSAS; GARCIA-CASAL, 2014). Contudo, a dependência aos fertilizantes químicos é dispendiosa, devido ao alto custo do produto e aos riscos associados ao uso indiscriminado desses insumos, como a eutrofização do solo e águas superficiais e subterrâneas e a emissão de gases de efeito estufa (DUNGAIT et al., 2012; MARKS et al., 2013).

Várias bactérias, tais como Azospirillum (MONTAÑEZ et al., 2009), Klebsiella (ARRUDA et al., 2013), Burkholderia (CHELIUS; TRIPLETT, 2001), Bacillus (BOMFIM et al., 2020) e Pseudomonas (PIROMYOU et al., 2011), têm sido identificadas como BPCP para milho por meio da fixação biológica de nitrogênio (FBN), solubilização de fosfato, produção de fitormônio e controle biológico de patógenos no solo. Tem sido relatado que a FBN pelas BPCP contribui com até $12-70 \%$ da absorção total de $\mathrm{N}$ em cultivo no campo ou $26,7 \mathrm{~kg} \mathrm{~N}^{-1}$ (70\% da absorção total de N) em milho (MONTAÑEZ et al., 2009), bem como aumento na captação de fósforo como resultado da inoculação 
de B. subtilis e Azospirillum brasilense, com um aumento de 100 e $54,6 \%$ na eficiência do uso do fósforo, respectivamente (PEREIRA et al., 2020).

Em condições de casa de vegetação, a inoculação de Bacillus pumilus S1r1, Klebsiella pneumoniae Fr1, Bacillus subtilis UPMB10 e Acinetobacter sp. S3r2 aumentou significativamente a absorção de N pela planta, a biomassa seca e o rendimento de espigas de milho (KUAN et al., 2016). Estes incrementos foram atribuídos, principalmente, à habilidade dessas linhagens em fixar nitrogênio, onde $B$. pumilus S1r1 é capaz de fixar até $304 \mathrm{mg} \mathrm{N}_{2}$ fixado planta $^{-1}$ na colheita da espiga e, possivelmente, outras habilidades, como produção de auxinas e solubilização de fosfato.

Em condições de campo, a inoculação das linhagens 40M e 42M de $A$. brasilense, individualmente ou em combinação, aumentou o rendimento de grãos de milho. Além destes incrementos, a inoculação de BPCP alterou a fisiologia das comunidades microbianas da rizosfera, modificando o número de bactérias fixadoras de nitrogênio microaerofílicas no estágio reprodutivo do milho, enquanto a fertilização nitrogenada modificou as comunidades de celulolíticos, nitrificantes e fixadores de nitrogênio microaerofílicas no estágio vegetativo do milho (DI SALVO et al., 2018). Este trabalho demonstra a importância de entendimento da ecologia microbiana da rizosfera, a qual pode ser usada para aumentar a resposta da inoculação da BPCP a fim de obter uma produção agrícola sustentável.

A inoculação de BPCPs em milho também pode influenciar na atividade e abundância de desnitrificadores na rizosfera, modificando a disponibilidade de carbono e nitrogênio (FLORIO et al., 2017). Com base em um experimento de campo e em câmara de crescimento, Florio et al. (2019) demonstraram que a inoculação de BPCPs pode aumentar a produção líquida de $\mathrm{N}_{2} \mathrm{O}$ em solos de terras agrícolas caracterizadas por alta limitação de $\mathrm{C}$, aumentando a abundância de redutores de $\mathrm{NO}_{2}^{-}$mais do que a abundância de redutores de $\mathrm{N}_{2} \mathrm{O}$. Por outro lado, a inoculação pode aumentar a capacidade do solo de agir como um reservatório de $\mathrm{N}_{2} \mathrm{O}$ para solos onde os desnitrificadores não são fortemente limitados por C. Sendo assim, a prática de inoculação deve ser avaliada em termos de possível aumento do rendimento das culturas, mas também possível emissão modificada de NOx, conjunto de gases com elevado potencial de efeito estufa (SÁNCHEZ-NAVARRO et al., 2020).

Além do uso das BPCPs como fertilizantes biológicos, estas bactérias também têm sido utilizadas para mitigar alguns estresses abióticos, como seca e salinidade. A linhagem Az19 de Azospirillum, por exemplo, é capaz de tolerar estresse osmótico e salino in vitro, e a inoculação de Az19 em mudas de milho permitiu uma maior tolerância ao estresse hídrico em relação às plantas não inoculadas (GARCIA et al., 2017). Já em relação à salinidade, a inoculação de $A$. brasilense Ab-V6 ou Rhizobium tropici CIAT 899, individualmente ou em combinação, foi capaz de aliviar os impactos negativos do estresse salino $(170 \mathrm{mM} \mathrm{NaCl})$ no milho, refletido na melhoria do crescimento das plantas e na expressão de enzimas de tolerância ao estresse e transcrição de genes (FUKAMI et al., 2018). Recentemente (ABDEL LATEF et al., 2020), demonstraram que a inoculação de Azospirillum lipoferum ou Azotobacter chroococcum melhorou significativamente os parâmetros de crescimento, pigmentos, $\mathrm{K}^{+}$, osmólitos, razão $\mathrm{K}^{+} / \mathrm{Na}^{+}$e a atividade de catalase, peroxidase e ascorbato peroxidase de plantas de milho afetadas por sal.

No Brasil, as linhagens Ab-V5 e Ab-V6 de A. brasilense são indicadas como inoculantes para milho, assim como para outras gramíneas, como trigo (FUKAMI et al., 2016) e pastagens com braquiária (HUNGRIA; NOGUEIRA; ARAUJO, 2016) e para coinoculação de leguminosas, como soja e feijão (HUNGRIA; NOGUEIRA; ARAUJO, 2013, 2015). Na safra de 2017 a 2018 foram comercializadas no Brasil cerca de 5 milhões de doses de inoculantes que contêm as cepas Ab-V5 e Ab-V6, e seu uso está se expandindo nos países vizinhos. Essas duas cepas são diferentes fenotipicamente, porém apresentam genomas altamente semelhantes que contêm genes relacionados à fixação de nitrogênio, síntese de fitormônios e adaptação ambiental (HUNGRIA; RIBEIRO; NOGUEIRA, 2018).

\section{Conclusões}

Existe alta diversidade entre as bactérias endofíticas na maioria dos ambientes e espécies estudadas, particularmente sob estresses abióticos. Estas bactérias apresentam um grande leque de mecanismos de promoção de crescimento, que costumam ser mais efetivos em plantas sob estresse ambiental. Estas diversidades indicam grande potencial para o uso de inoculação destes micro-organismos para a promoção da produtividade ou redução do uso de insumos como fertilizantes, como pode ser exemplificado pelo uso atual desta prática na cultura do milho

\section{Agradecimentos}

Os autores F. J. C. F. e M. J. G. A. agradecem à Coordenação de Aperfeiçoamento de Pessoal de Nível Superior (CAPES), J. J. M à Fundação de Amparo à Ciência e Tecnologia do Estado de Pernambuco (FACEPE) (IBPG-0888-5.01/16) e M. A. L. J. ao Conselho Nacional de Desenvolvimento Científico e Tecnológico (CNPq, Brasil) (306388/2016-2) e FACEPE (BPV-0005-5.010/18 e BPV-0008-5.01/19). Todos os autores agradecem à CAPES (Código Financeiro 001), CNPq (401896/2013-7 e 483287/2013-0) e FACEPE (APQ-0453-5.01/15) pelo suporte financeiro.

\section{Referências}

Aamir, M. et al. Co-inoculation with rhizobium and plant growth promoting rhizobacteria (pgpr) for inducing salinity tolerance in mung bean under field condition of semi-arid climate. Asian Journal of Agriculture and Biology, Pakistan, v. 1, n. 1, p. 17-22, 2013.

Abdel Latef, A. A. H. et al. Inoculation with Azospirillum lipoferum or Azotobacter chroococcum reinforces maize growth by improving physiological activities under saline conditions. Journal of Plant Growth Regulation, United States, v. 39, p. 1293-1306, 2020.

Afzal, I. et al. Plant beneficial endophytic bacteria: mechanisms, diversity, host range and genetic determinants. Microbiological Research, Germany, v. 221, p. 36-49, 2019. 
Afzal, I. et al. Plant growth-promoting potential of endophytic bacteria isolated from roots of wild Dodonaea viscosa L. Plant Growth Regulation, Netherlands, v. 81, n. 3, p. 399-408, 2017.

Aloo, B. N.; Makumba, B. A.; Mbega, E. R. The potential of Bacilli rhizobacteria for sustainable crop production and environmental sustainability. Microbiological Research, Germany, v. 219, p. 26-39, 2019.

Amin, A.; Latif, Z. Screening of mercury-resistant and indole-3-acetic acid producing bacterial-consortium for growth promotion of Cicer arietinum L. Journal of Basic Microbiology, Germany, v. 57, n. 3, p. 204-217, 2017.

Araújo, M. D. S. B.; Sampaio, E. V. S. B.; Schaefer, C. E. R. Phosphorus desorption affected by drying and wetting cycles in Ferralsols and Luvisols of Brazilian Northeast. Archives of Agronomy and Soil Science, United Kingdom, v. 63, n. 2, p. 242-249, 2017.

Archana, G.; Buch, A.; Kumar, G. N. Pivotal role of organic acid secretion by rhizobacteria in plant growth promotion. In: Satyanarayana, T., Johri, B. N., Anil P. Microorganisms in sustainable agriculture and biotechnology. Springer Netherlands, 2012. p. 35-53. Dordrecht: Springer Netherlands, 2012. p. 35-53.

Armendariz, A. L. et al. Impact of double inoculation with Bradyrhizobium japonicum E109 and Azospirillum brasilense Az39 on soybean plants grown under arsenic stress. Plant Physiology and Biochemistry, Netherlands, v. 138, p. 26-35, 2019.

Arruda, L. et al. Screening of rhizobacteria isolated from maize (Zea mays L.) in Rio Grande do Sul State (South Brazil) and analysis of their potential to improve plant growth. Applied Soil Ecology, Netherlands, v. 63, p. 15-22, 2013.

Arshad, M. et al. Comparison of rhizospheric bacterial populations and growth promotion of AVP1 transgenic and non-transgenic cotton by bacterial inoculations. Journal of Animal and Plant Sciences, Lahore, v. 26, n. 5, p. 1284-1290, 2016.

Arzanesh, M. H. et al. Wheat (Triticum aestivum L.) growth enhancement by Azospirillum sp. under drought stress. World Journal of Microbiology \& Biotechnology, Oxford, v. 27, n. 2, p. 197-205, 2011.

Banik, A.; Mukhopadhaya, S. K.; Dangar, T. K. Characterization of $\mathrm{N}_{2}$-fixing plant growth promoting endophytic and epiphytic bacterial community of Indian cultivated and wild rice (Oryza spp.) genotypes. Planta, Berlin, v. 243, n. 3, p. 799-812, 2016.

Barnawal, D. et al. ACC deaminase-containing Arthrobacter protophormiae induces $\mathrm{NaCl}$ stress tolerance through reduced $\mathrm{ACC}$ oxidase activity and ethylene production resulting in improved nodulation and mycorrhization in Pisum sativum. Journal of Plant Physiology, Netherlands, v. 171, n. 11, p. 884-894, 2014.

Barnawal, D. et al. Plant growth-promoting rhizobacteria enhance wheat salt and drought stress tolerance by altering endogenous phytohormone levels and TaCTR1/TaDREB2 expression. Physiologia Plantarum, United Kingdom, v. 161, n. 4, p. 502-514, 2017.

Basu, S.; Rabara, R.; Negi, S. Towards a better greener future - an alternative strategy using biofertilizers. I: Plant growth promoting bacteria. Plant Gene, Netherlands, v. 12, p. 43-49, 2017.

Batista, B. D. et al. Screening of tropically derived, multi-trait plant growth- promoting rhizobacteria and evaluation of corn and soybean colonization ability. Microbiological Research, Germany, v. 206, p. 33-42, 2018.

Beutler, A. N. et al. Effect of nitrogen-fixing bacteria on grain yield and development of flooded irrigated rice. Revista Caatinga, Mossoró, v. 29, n. 1, p. 11-17, 2016.
Bhattacharyya, P. N.; Jha, D. K. Plant growth-promoting rhizobacteria (PGPR): Emergence in agriculture. World Journal of Microbiology \& Biotechnology, Oxford, v. 28, n. 4, p. 1327-1350, 2012.

Bizos, G. et al. The role of microbial inoculants on plant protection, growth stimulation, and crop productivity of the olive tree (Olea europea 1.). Plants, Switzerland, v. 9, n. 6, p. 1-17, 2020.

Bolhuis, $H$. et al. Horizontal transfer of the nitrogen fixation gene cluster in the cyanobacterium Microcoleus chthonoplastes. The ISME Journal, United Kingdom, v. 4, n. 1, p. 121-130, 2010.

Bomfim, C. S. G. et al. Endophytic bacteria naturally inhabiting commercial maize seeds occupy different niches and are efficient plant growth-promoting agents. Symbiosis, United States, v. 81, n. 3, p. 255-269, 2020.

Brasil. Instrução Normativa SDA no13, de 24 de março de 2011. Aprova as normas sobre especificações, garantias, registro, embalagem e rotulagem dos inoculantes destinados à agricultura, bem como as relações dos micro-organismos autorizados e recomendados para produção de inoculantes no Brasil. Diário Oficial da República Federativa do Brasil, Brasília, DF, 25 mar. 2011. Seção 1, No. 58, p. 3-7.

Bulgarelli, D. et al. Structure and functions of the bacterial microbiota of plants. Annual Review of Plant Biology, United States, v. 64, p. 807-838, 2013.

Burr, T. J.; Caesar, A. Beneficial plant bacteria. Critical Reviews in Plant Sciences, United Kingdom, v. 2, n. 1, p. 1-20, 1984.

Canfield, D. E.; Glazer, A. N.; Falkowski, P. G. The evolution and future of earth's nitrogen cycle. Science, United States, v. 330, n. 6001, p. 192-196, 2010.

Cardoso, I. C. M. et al. Ocorrência de bactérias endofíticas do gênero Azospirillum em arroz irrigado no estado de Santa Catarina. Revista de Ciências Agroveterinárias, Lages, v. 9, n. 2, p. 178-186, 2010.

Carvalho, E. X. et al. The ${ }^{15} \mathrm{~N}$ natural abundance technique to assess the potential of biological nitrogen fixation (BNF) in some important $\mathrm{C}_{4}$ grasses. Australian Journal of Crop Science, Lismore, v. 11, n. 12, p. 1559-1564, 2017.

Cassán, F. et al. Azospirillum brasilense Az39 and Bradyrhizobium japonicum E109, inoculated singly or in combination, promote seed germination and early seedling growth in corn (Zea mays L.) and soybean (Glycine max L.). European Journal of Soil Biology, France, v. 45, n. 1, p. 28-35, 2009.

Chandra, D. et al. Field performance of bacterial inoculants to alleviate water stress effects in wheat (Triticum aestivum L.). Plant and Soil, Netherlands, v. 441, n. 1-2, p. 261-281, 2019.

Chaudhary, H. J. et al. Genetic diversity of endophytic diazotrophs of the wild rice, oryza alta and identification of the new diazotroph, acinetobacter oryzae sp. nov. Microbial Ecology, United States, v. 63, n. 4 , p. $813-821,2012$.

Chelius, M. K.; Triplett, E. W. The diversity of archaea and bacteria in association with the roots of Zea mays L. Microbial Ecology, United States, v. 41, n. 3, p. 252-263, 2001.

Cherif-Silini, H. et al. Durum wheat stress tolerance induced by endophyte Pantoea agglomerans with genes contributing to plant functions and secondary metabolite arsenal. International Journal of Molecular Sciences, Switzerland, v. 20, n. 16, 2019.

Cherif-Silini, H. et al. Phylogenetic and plant-growth-promoting characteristics of Bacillus isolated from the wheat rhizosphere. Annals of Microbiology, Germany, v. 66, n. 3, p. 1087-1097, 2016.

$\mathrm{Chi}$, F. et al. Ascending migration of endophytic rhizobia, from roots to leaves, inside rice plants and assessment of benefits to rice growth 
physiology. Applied and Environmental Microbiology, United States, v. 71, n. 11, p. 7271-7278, 2005.

Chibeba, A. M. et al. Towards sustainable yield improvement: field inoculation of soybean with Bradyrhizobium and co-inoculation with Azospirillum in Mozambique. Archives of Microbiology, Germany, v. 202, p. 2579-2590, 2020.

Cusack, D. F.; Silver, W.; Mcdowell, W. H. Biological nitrogen fixation in two tropical forests: Ecosystem-level patterns and effects of nitrogen fertilization. Ecosystems, New York, v. 12, n. 8, p. 1299-1315, 2009.

Dar, T. A. et al. Jasmonates counter plant stress: a review. Environmental and Experimental Botany, Netherlands, v. 115, p. 49-57, 2015.

Di Salvo, L. P. et al. Plant growth-promoting rhizobacteria inoculation and nitrogen fertilization increase maize (Zea mays L.) grain yield and modified rhizosphere microbial communities. Applied Soil Ecology, Netherlands, v. 126, p. 113-120, 2018.

Domenech, J. et al. Combined application of the biological product LS213 with Bacillus, Pseudomonas or Chryseobacterium for growth promotion and biological control of soil-borne diseases in pepper and tomato. BioControl, Netherlands, v. 51, n. 2, p. 245-258, 2006.

Dungait, J. A. et al. Advances in the understanding of nutrient dynamics and management in UK agriculture. Science of the Total Environment, Netherlands, v. 434, p. 39-50, 2012.

Edwards, J. et al. Structure, variation, and assembly of the root-associated microbiomes of rice. Proceedings of the National Academy of Sciences of the United States of America, United States, v. 112, n. 8, p. E911-E920, 2015.

Emami, S. et al. Effect of rhizospheric and endophytic bacteria with multiple plant growth promoting traits on wheat growth. Environmental Science and Pollution Research International, Germany, v. 26, n. 19, p. 19804-19813, 2019.

Fernandes-Júnior, P. I. et al. The ressurection plant Tripogon spicatus (Poaceae) harbors a diversity of plant growth promoting bacteria in Northeastern Brazilian caatinga. Revista Brasileira de Ciência do Solo, Viçosa, v. 39, p. 993-1002, 2015.

Florio, A. et al. Soil C and N statuses determine the effect of maize inoculation by plant growth-promoting rhizobacteria on nitrifying and denitrifying communities. Scientific Reports, London, v. 7, n. 1, p. $8411,2017$.

Florio, A. et al. The responses of $\mathrm{NO}_{2}^{-}-$and $\mathrm{N}_{2} \mathrm{O}$-reducing bacteria to maize inoculation by the PGPR Azospirillum lipoferum CRT1 depend on carbon availability and determine soil gross and net $\mathrm{N}_{2} \mathrm{O}$ production. Soil Biology \& Biochemistry, United Kingdom, v. 136, p. 107524, 2019.

Fukami, J. et al. Accessing inoculation methods of maize and wheat with Azospirillum brasilense. AMB Express, Heidelberg, v. 6, n. 1, p. 1-13, 2016.

Fukami, J. et al. Co-inoculation of maize with Azospirillum brasilense and Rhizobium tropici as a strategy to mitigate salinity stress. Functional Plant Biology, Collingwood, v. 45, n. 3, p. 328-339, 2018.

Fukami, J.; Cerezini, P.; Hungria, M. Azospirillum: benefits that go far beyond biological nitrogen fixation. AMB Express, Heidelberg, v. 8 , n. 1, p. 73, 2018.

Garcia, J. E. et al. In vitro PGPR properties and osmotic tolerance of different Azospirillum native strains and their effects on growth of maize under drought stress. Microbiological Research, Germany, v. 202, p. 21-29, 2017.

Glick, B. R. et al. ACC deaminase is central to the functioning of plant growth promoting rhizobacteria. In: KANELLIS, A. K. et al. (Ed.).
Biology and biotechnology of the plant hormone Ethylene II. Dordrecht: Springer, 1999.

Gonzalez, A. M. et al. Diversity of bacterial communities related to the nitrogen cycle in a coastal tropical bay. Molecular Biology Reports, Netherlands, v. 39, n. 4, p. 3401-3407, 2012.

Granada, C. E. et al. Is phosphate solubilization the forgotten child of plant growth-promoting rhizobacteria? Frontiers in Microbiology, Lausanne, v. 9, p. 2054, 2018.

Grobelak, A.; Napora, A.; Kacprzak, M. Using plant growth-promoting rhizobacteria (PGPR) to improve plant growth. Ecological Engineering, Amsterdam, v. 84, p. 22-28, 2015.

Grover, M.; Nain, L.; Saxena, A. K. Comparision between Bacillus subtilis RP24 and its antibiotic-defective mutants. World Journal of Microbiology \& Biotechnology, Oxford, v. 25, n. 8, p. 1329-1335, 2009.

Gutjahr, C.; Paszkowski, U. Weights in the balance: Jasmonic acid and Salicylic acid signaling in root-biotroph interactions. Molecular Plant-Microbe Interactions, St. Paul, v. 22, n. 7, p. 763-772, 2009.

Hahn, L. et al. Rhizobial inoculation, alone or coinoculated with Azospirillum brasilense, promotes growth of wetland rice. Revista Brasileira de Ciência do Solo, Viçosa, v. 40, p. e0160006, 2016.

Hardoim, P. R. et al. The hidden world within plants: ecological and evolutionary considerations for defining functioning of microbial endophytes. Microbiology and Molecular Biology Reviews, Washington, v. 79, n. 3, p. 293-320, 2015.

Harman, G. E.; Uphoff, N. Symbiotic root-endophytic soil microbes improve crop productivity and provide environmental benefits. Scientifica, Cairo, v. 2019, p. 9106395, 2019.

Hashem, A.; Tabassum, B.; FATHI ABD_ALLAH, E. Bacillus subtilis: a plant-growth promoting rhizobacterium that also impacts biotic stress. Saudi Journal of Biological Sciences, Riyadh, v. 26, n. 6, p. 1291-1297, 2019.

Hungria, M.; Nogueira, M. A.; Araujo, R. S. Co-inoculation of soybeans and common beans with rhizobia and azospirilla: strategies to improve sustainability. Biology and Fertility of Soils, Berlin, v. 49, n. 7, p. 791-801, 2013.

Hungria, M.; Nogueira, M. A.; Araujo, R. S. Inoculation of Brachiaria spp. with the plant growth-promoting bacterium Azospirillum brasilense: An environment-friendly component in the reclamation of degraded pastures in the tropics. Agriculture, Ecosystems \& Environment, Amsterdam, v. 221, p. 125-131, 2016.

Hungria, M.; Nogueira, M. A.; Araujo, R. S. Soybean seed co-inoculation with Bradyrhizobium; spp. and Azospirillum brasilense: a new biotechnological tool to improve yield and sustainability. American Journal of Plant Sciences, Irvine, Calif, v. 6, n. 6, p. 811-817, 2015.

Hungria, M.; Ribeiro, R. A.; Nogueira, M. A. Draft genome sequences of Azospirillum brasilense strains Ab-V5 and Ab-V6, commercially used in inoculants for grasses and legumes in Brazil. Genome Announcements, Washington, v. 6, n. 20, 2018.

Hussain, A. et al. Effect of IAA on in vitro growth and colonization of Nostoc in plant roots. Frontiers of Plant Science, Lausanne, v. 6, p. $46,2015$.

Igiehon, N. O.; Babalola, O. O.; Aremu, B. R. Genomic insights into plant growth promoting rhizobia capable of enhancing soybean germination under drought stress. BMC Microbiology, London, v. 19, n. 1, p. 159, 2019.

Ikeda, A. C. et al. Bioprospecting of elite plant growth-promoting bacteria for the maize crop. Acta Scientiarum. Agronomy, Maringá, v. 42, n. 1, p. 44364, 2020. 
Ji, S. H.; Gururani, M. A.; Chun, S. C. Isolation and characterization of plant growth promoting endophytic diazotrophic bacteria from Korean rice cultivars. Microbiological Research, Germany, v. 169, n. 1, p. 83-98, 2014.

Jochum, M. D. et al. Bioprospecting Plant Growth-Promoting Rhizobacteria That Mitigate Drought Stress in Grasses. Frontiers in Microbiology, Lausanne, v. 10, p. 2106, 2019.

Kakar, K. U. et al. Rhizosphere-associated Alcaligenes and Bacillus strains that induce resistance against blast and sheath blight diseases, enhance plant growth and improve mineral content in rice. Journal of Applied Microbiology, United Kingdom, v. 124, n. 3, p. 779-796, 2018.

Kaul, S.; Sharma, T.; Dhar, M. K. "Omics” tools for better understanding the plant-endophyte interactions. Frontiers of Plant Science, Lausanne, v. 7, p. 955, 2016.

Khan, A. L. et al. Indole acetic acid and ACC deaminase from endophytic bacteria improves the growth of Solanum lycopersicum. Electronic Journal of Biotechnology, Chile, v. 21, p. 58-64, 2016.

Khatabi, B. et al. Plant-microbe symbiosis: what has proteomics taught us? Proteomics, New York, v. 19, n. 16, p. e1800105, 2019.

Kloepper, J. W. Plant growth-promoting rhizobacteria on radishes. In: CONFERENCE ON PLANT PATHOGENIC BACTER, STATION DE PATHOLOGIE VEGETALE ET PHYTOBACTERIOLOGIE, 4., 1978, Angers, France. Proceedings... France: INRA, 1978. p.879-882.

Kour, D. et al. Microbial biofertilizers: bioresources and eco-friendly technologies for agricultural and environmental sustainability. Biocatalysis and Agricultural Biotechnology, Atlanta, v. 23, p. 101487, 2020.

Kuan, K. B. et al. Plant growth-promoting rhizobacteria inoculation to enhance vegetative growth, nitrogen fixation and nitrogen remobilisation of maize under greenhouse conditions. PLoS One, San Francisco, v. 11, n. 3, p. e0152478-e0152478, 2016.

Kuźniar, A.; Włodarczyk, K.; Wolińska, A. Agricultural and other biotechnological applications resulting from trophic plant-endophyte interactions. Agronomy, Basel, Switzerland, v. 9, n. 12, 2019.

Leite, R. D. C. et al. Productivity increase, reduction of nitrogen fertiliser use and drought-stress mitigation by inoculation of Marandu grass (Urochloa brizantha) with Azospirillum brasilense. Crop \& Pasture Science, Collingwood, v. 70, n. 1, p. 61-67, 2019.

$\mathrm{Li}, \mathrm{X}$. et al. Mechanisms for solubilization of various insoluble phosphates and activation of immobilized phosphates in different soils by an efficient and salinity-tolerant aspergillus niger strain An2. Applied Biochemistry and Biotechnology, United States, v. 175 , n. 5, p. $2755-2768,2015$.

$\mathrm{Li}, \mathrm{H}$. B. et al. Genetic diversity of nitrogen-fixing and plant growth promoting Pseudomonas Species isolated from sugarcane rhizosphere. Frontiers in Microbiology, Lausanne, v. 8, p. 1268, 2017 a.

Li, Y. et al. Colonization and maize growth promotion induced by phosphate solubilizing bacterial isolates. International Journal of Molecular Sciences, Switzerland, v. 18, n. 7, p. 1253-1253, 2017b.

Lian, B. et al. Effects of salicylic acid on the development and root nodulation of soybean seedlings. Journal of Agronomy \& Crop Science, Berlin, v. 185, n. 3, p. 187-192, 2000.

Lin, Y. et al. Influence of plant growth-promoting rhizobacteria on corn growth under different fertility sources. Communications in Soil Science and Plant Analysis, New York, v. 49, n. 10, p. 1239-1255, 2018.
Liu, H. et al. Inner plant values: diversity, colonization and benefits from endophytic bacteria. Frontiers in Microbiology, Lausanne, v. 8, n. 2552, 2017.

Longhini, V. Z. et al. Inoculation of diazotrophic bacteria and nitrogen fertilization in topdressing in irrigated corn. Revista Caatinga, Mossoró, v. 29, n. 2, p. 338-347, 2016.

Lowman, S. et al. Strategies for enhancement of switchgrass (Panicum virgatum L.) performance under limited nitrogen supply based on utilization of N-fixing bacterial endophytes. Plant and Soil, Netherlands, v. 405, n. 1, p. 47-63, 2016.

Lyu, Y. et al. Major crop species show differential balance between root morphological and physiological responses to variable phosphorus supply. Frontiers of Plant Science, Lausanne, v. 7, p. 1939-1939, 2016.

Machado, V. et al. Bacteria as biocontrol agents of plant parasitic nematodes. Oecologia Australis, Rio de Janeiro, v. 16, n. 2, p. 165-182, 2012.

Magnani, G. S. et al. Culture-independent analysis of endophytic bacterial communities associated with Brazilian sugarcane. Genetics and Molecular Research, Ribeirão Preto, v. 12, n. 4, p. 4549-4558, 2013.

Mahmud, K. et al. Current progress in nitrogen fixing plants and microbiome research. Plants, Switzerland, v. 9, n. 1, 2020.

Marag, P. S.; Suman, A. Growth stage and tissue specific colonization of endophytic bacteria having plant growth promoting traits in hybrid and composite maize (Zea mays L.). Microbiological Research, Germany, v. 214, p. 101-113, 2018.

Marks, B. B. et al. Biotechnological potential of rhizobial metabolites to enhance the performance of Bradyrhizobium spp. and Azospirillum brasilense inoculants with soybean and maize. AMB Express, Heidelberg, v. 3, n. 1, p. 21-21, 2013.

Marquez-Santacruz, H. A. et al. Diversity of bacterial endophytes in roots of Mexican husk tomato plants (Physalis ixocarpa) and their detection in the rhizosphere. Genetics and Molecular Research, Ribeirão Preto, v. 9, n. 4, p. 2372-2380, 2010.

Mccue, P. et al. A model for enhanced pea seedling vigour following low $\mathrm{pH}$ and salicylic acid treatments. Process Biochemistry, Barking, Essex, England, v. 35, n. 6, p. 603-613, 2000.

Mendes, G. O. et al. Mechanisms of phosphate solubilization by fungal isolates when exposed to different P sources. Annals of Microbiology, Germany, v. 64, n. 1, p. 239-249, 2014.

Mendonça, J. J. et al. Avaliação in vitro dos mecanismos de promoção de crescimento das bactérias isoladas do capim pangolão. In: CONGRESO LATINOAMERICANO DE LA CIENCIA DEL SUELO - CLACS, 22., 2019, Montevideo. Anales.... Montevideo: Easy Planners, 2019. CD-ROM

Menéndez, E.; Paço, A. Is the application of plant probiotic bacterial consortia always beneficial for plants? Exploring synergies between rhizobial and non-rhizobial bacteria and their effects on agroeconomically valuable crops. Life Chicago, v. 10, n. 3, p. 24, 2020.

Montañez, A. et al. Biological nitrogen fixation in maize (Zea mays L.) by ${ }^{15} \mathrm{~N}$ isotope-dilution and identification of associated culturable diazotrophs. Biology and Fertility of Soils, Berlin, v. 45, n. 3, p. 253-263, 2009

Moretti, L. G. et al. Effects of growth-promoting bacteria on soybean root activity, plant development, and yield. Agronomy Journal, United States, v. 112, n. 1, p. 418-428, 2020.

Narayanasamy, S.; Thangappan, S.; Uthandi, S. Plant growth-promoting Bacillus sp. cahoots moisture stress alleviation in rice genotypes by 
triggering antioxidant defense system. Microbiological Research, Germany, v. 239, p. 126518, 2020.

Niehus, R. et al. The evolution of siderophore production as a competitive trait. Evolution, Sweden, v. 71, n. 6, p. 1443-1455, 2017.

Oliveira-Longatti, S. M. et al. The culture medium volume and the inoculation method should be considered in semi-quantitative screening of calcium phosphate solubilization by bacteria. Acta Scientiarum. Agronomy, Maringá, v. 42, p. e44332, 2020.

Paredes, I. et al. Agricultural and urban delivered nitrate pollution input to Mediterranean temporary freshwaters. Agriculture, Ecosystems and Environment, Amsterdam, v. 294, p. 106859, 2020.

Patel, J. K.; Archana, G. Diverse culturable diazotrophic endophytic bacteria from Poaceae plants show cross-colonization and plant growth promotion in wheat. Plant and Soil, Netherlands, v. 417, n. 1-2, p. 99-116, 2017.

Pereira, N. C. M. et al. Corn yield and phosphorus use efficiency response to phosphorus rates associated with plant growth promoting bacteria. Frontiers in Environmental Science, Lausanne, v. 8, p. 40, 2020.

Pereira, W. et al. Sugarcane productivity as a function of nitrogen fertilization and inoculation with diazotrophic plant growth-promoting bacteria. Sugar Tech, India, v. 21, n. 1, p. 71-82, 2019.

Perez-Montano, F. et al. Plant growth promotion in cereal and leguminous agricultural important plants: from microorganism capacities to crop production. Microbiological Research, Germany, v. 169, n. 5-6, p. 325-336, 2014.

Pieterse, C. M. J.; De Jonge, R.; Berendsen, R. L. The soil-borne supremacy. Trends in Plant Science, Kidlington, Oxford, v. 21, n. 3, p. 171-173, 2016.

Piromyou, P. et al. Effect of plant growth promoting rhizobacteria (PGPR) inoculation on microbial community structure in rhizosphere of forage corn cultivated in Thailand. European Journal of Soil Biology, France, v. 47, n. 1, p. 44-54, 2011.

Puente, M. L. et al. Improvement of soybean grain nutritional quality under foliar inoculation with Azospirillum brasilense strain Az39. Symbiosis, United States, v. 77, n. 1, p. 41-47, 2019.

Puente, M. L. et al. The benefits of foliar inoculation with Azospirillum brasilense in soybean are explained by an auxin signaling model. Symbiosis, United States, v. 76, n. 1, p. 41-49, 2018.

Puri, A.; Padda, K. P.; Chanway, C. P. In vitro and in vivo analyses of plant-growth-promoting potential of bacteria naturally associated with spruce trees growing on nutrient-poor soils. Applied Soil Ecology, Netherlands, v. 149, p. 103538, 2020.

Ramakrishna, W.; Yadav, R.; Li, K. Plant growth promoting bacteria in agriculture: two sides of a coin. Applied Soil Ecology, Netherlands, v. 138 , p. 10-18, 2019.

Ranum, P.; Peña-Rosas, J. P.; Garcia-Casal, M. N. Global maize production, utilization, and consumption. Annals of the New York Academy of Sciences, New York, v. 1312, p. 105-112, 2014.

Reed, S. C.; Cleveland, C. C.; Townsend, A. R. Functional ecology of free-living nitrogen fixation: a contemporary perspective. Annual Review of Ecology Evolution and Systematics, Palo Alto, v. 42, p. 489-512, 2011.

Ren, G. et al. Response of leaf endophytic bacterial community to elevated $\mathrm{CO} 2$ at different growth stages of rice plant. Frontiers in Microbiology, Lausanne, v. 6, p. 855-855, 2015.

Rodrigues, A. A. et al. Isolation and prospection of diazotrophic rhizobacteria associated with sugarcane under organic management. Anais da Academia Brasileira de Ciências, Rio de Janeiro, v. 90, n. 4, p. 3813-3829, 2018.
Rondina, A. B. L. et al. Changes in root morphological traits in soybean co-inoculated with Bradyrhizobium spp. and Azospirillum brasilense or treated with A. brasilense exudates. Biology and Fertility of Soils, Berlin, v. 56, p. 537-549, 2020.

Rotaru, V. I.; Risnoveanu, L. Interactive effects of plant growth-promoting rhizobacteria and phosphates sources on growth and phosphorus nutrition of soybean under moderate drought. Notulae Botanicae Horti Agrobotanici Cluj-Napoca, Cluj-Napoca, Romania, v. 47, n. 3, p. 872-880, 2019.

Saghafi, D. et al. An overview on improvement of crop productivity in saline soils by halotolerant and halophilic PGPRs. 3 Biotech, Berlin, v. 9, p. 261, 2019.

Sala, V. M. R. et al. Interaction of new diazotrophic endophytic bacteria and nitrogen fertilization on wheat crop under field conditions. Revista Brasileira de Ciência do Solo, Viçosa, v. 32, n. 3, p. 1099-1106, 2008.

Sánchez-Navarro, V. et al. A comparative greenhouse gas emissions study of legume and non-legume crops grown using organic and conventional fertilizers. Scientia Horticulturae, Amsterdam, v. 260, p. 108902, 2020.

Santos, M. L. D. et al. Benefits associated with the interaction of endophytic bacteria and plants. Brazilian Archives of Biology and Technology, Curitiba, v. 61, p. e18160431, 2018.

Santoyo, G. et al. Plant growth-promoting bacterial endophytes. Microbiological Research, Germany, v. 183, p. 92-99, 2016.

Sarkar, A. et al. A halotolerant Enterobacter sp. displaying ACC deaminase activity promotes rice seedling growth under salt stress. Research in Microbiology, France, v. 169, n. 1, p. 20-32, 2018.

Sarkar, A.; Reinhold-Hurek, B. Transcriptional profiling of nitrogen fixation and the role of NifA in the diazotrophic endophyte Azoarcus sp. strain BH72. PLoS One, San Francisco, v. 9, n. 2, p. 12-12, 2014.

Saxena, A. K. et al. Bacillus species in soil as a natural resource for plant health and nutrition. Journal of Applied Microbiology, United Kingdom, v. 128, n. 6, p. 1583-1594, 2019.

Sessitsch, A. et al. Functional characteristics of an endophyte community colonizing rice roots as revealed by metagenomic analysis. Molecular Plant-Microbe Interactions, St. Paul, v. 25, n. 1, p. 28-36, 2012.

Shaharoona, B. et al. Effectiveness of various Pseudomonas spp. and Burkholderia caryophylli containing ACC-deaminase for improving growth and yield of wheat (Triticum aestivum L.). Journal of Microbiology and Biotechnology, Korea, v. 17, n. 8, p. 1300-1307, 2007.

Shaharoona, B.; Arshad, M.; Zahir, Z. A. Effect of plant growth promoting rhizobacteria containing ACC-deaminase on maize (Zea mays L.) growth under axenic conditions and on nodulation in mung bean (Vigna radiata L.). Letters in Applied Microbiology, Oxford, v. 42, n. 2, p. 155-159, 2006.

Shao, J. et al. Contribution of indole-3-acetic acid in the plant growth promotion by the rhizospheric strain Bacillus amyloliquefaciens SQR9. Biology and Fertility of Soils, Berlin, v. 51, n. 3, p. 321-330, 2015.

Shen, F. T. et al. Screening of rice endophytic biofertilizers with fungicide tolerance and plant growth-promoting characteristics. Sustainability, Basel, Switzerland, v. 11, n. 4, p. 1133, 2019.

Siddiqui, I. A.; Shaukat, S. S. Mixtures of plant disease suppressive bacteria enhance biological control of multiple tomato pathogens. Biology and Fertility of Soils, Berlin, v. 36, n. 4, p. 260-268, 2002.

Siddiqui, Z. A.; Baghel, G.; Akhtar, M. S. Biocontrol of Meloidogyne javanica by Rhizobium and plant growth-promoting rhizobacteria 
on lentil. World Journal of Microbiology \& Biotechnology, Oxford, v. 23, n. 3, p. 435-441, 2007.

Silva, M. D. O. et al. Isolamento e prospecção de bactérias endofíticas e epifíticas na cana-de-açúcar em áreas com e sem cupinicida. Revista Brasileira de Ciência do Solo, Viçosa, v. 36, p. 1113-1122, 2012.

Silva, M. L. R. B. et al. Diversidade e potencial de solubilização de fosfato in vitro por bactérias endofíticas associadas à cultura da palma forrageira (Opuntia e Nopalea) em Pernambuco. Pesquisa Agropecuária Pernambucana, Recife, v. 19, n. 2, p. 85-88, 2014.

Silva, T. F. et al. Non-symbiotic diazotrophic bacteria and the rooting of olive semi-hardwood cuttings (Olea europaea L.). Ciência Florestal, Santa Maria, v. 27, n. 1, p. 61-71, 2017.

Silveira, A. P. D. D. et al. Nitrogen metabolism and growth of wheat plant under diazotrophic endophytic bacteria inoculation. Applied Soil Ecology, Netherlands, v. 107, p. 313-319, 2016.

Silveira, A. P. D. et al. Exploitation of new endophytic bacteria and their ability to promote sugarcane growth and nitrogen nutrition. Antonie van Leeuwenhoek. International Journal of General and Molecular Microbiology, Netherlands, v. 112, n. 2, p. 283-295, 2019.

Sousa, C. A. et al. Evaluation methods used for phosphate-solubilizing bacteria. African Journal of Biotechnology, Nigeria, v. 15, n. 34, p. 1796-1805, 2016.

Souza, E. M. et al. Does the nitrogen application associated with Azospirillum brasilense inoculation influence corn nutrition and yield? Revista Brasileira de Engenharia Agrícola e Ambiental, Campina Grande, v. 23, n. 1, p. 53-59, 2019.

Stirling, G. R. et al. Pasteuria, a bacterial parasite of plant-parasitic nematodes: its occurrence in Australian sugarcane soils and its role as a biological control agent in naturally-infested soil. Australasian Plant Pathology, New Zealand, v. 46, p. 563-569, 2017.

Sturz, A. V.; Nowak, J. Endophytic communities of rhizobacteria and the strategies required to create yield enhancing associations with crops. Applied Soil Ecology, Netherlands, v. 15, n. 2, p. 183-190, 2000.

Sulochana, M. B. et al. Siderophore as a potential plant growthpromoting agent produced by Pseudomonas aeruginosa JAS-25. Applied Biochemistry and Biotechnology, United States, v. 174, n. 1, p. 297-308, 2014.

Taulé, C. et al. Endophytic colonization of sugarcane (Saccharum officinarum) by the novel diazotrophs Shinella sp. UYSO24 and Enterobacter sp. UYSO10. Plant and Soil, Netherlands, v. 403, n. 1-2, p. 403-418, 2016.

Taulé, C. et al. The contribution of nitrogen fixation to sugarcane (Saccharum officinarum L.), and the identification and characterization of part of the associated diazotrophic bacterial community. Plant and Soil, Netherlands, v. 356, n. 1-2, p. 35-49, 2012.

Theisen, G. et al. The birth of a new cropping system: towards sustainability in the sub-tropical lowland agriculture. Field Crops Research, Netherlands, v. 212, p. 82-94, 2017.

Tian, B.; Yang, J.; Zhang, K. Q. Bacteria used in the biological control of plant-parasitic nematodes: Populations, mechanisms of action, and future prospects. FEMS Microbiology Ecology, United Kingdom, v. 61, n. 2, p. 197-213, 2007.

Torres, M. et al. Growth promotion on horticultural crops and antifungal activity of Bacillus velezensis XT1. Applied Soil Ecology, Netherlands, v. 150, p. 103453, 2020.

Valdez-Nuñez, R. A. et al. PGPR characterization of non-nodulating bacterial endophytes from root nodules of Vigna unguiculata (L.) Walp. In: Zúñiga-Dávila, D. et al. (Ed.). Microbial probiotics for agricultural systems. Advances in agronomic use. Zurique: Springer International Publishing, 2019. p. 111-126.

Verma, S. K.; White, J. F. Indigenous endophytic seed bacteria promote seedling development and defend against fungal disease in browntop millet (Urochloa ramosa L.). Journal of Applied Microbiology, United Kingdom, v. 124, n. 3, p. 764-778, 2018.

White, J. F. et al. Review: endophytic microbes and their potential applications in crop management. Pest Management Science, United States, v. 75, n. 10, p. 2558-2565, 2019.

Win, K. T. et al. The ACC deaminase expressing endophyte Pseudomonas spp. Enhances $\mathrm{NaCl}$ stress tolerance by reducing stress-related ethylene production, resulting in improved growth, photosynthetic performance, and ionic balance in tomato plants. Plant Physiology and Biochemistry, Netherlands, v. 127, p. 599-607, 2018.

Wood, J. L. et al. Microbial community dynamics in the rhizosphere of a cadmium hyper-accumulator. Scientific Reports, London, v. 6, p. 1-10, 2016.

Xia, Y. et al. Recent advances in control technologies for non-point source pollution with nitrogen and phosphorous from agricultural runoff: current practices and future prospects. Applied Biological Chemistry, Korea, v. 63, n. 1, 2020.

Xing, Y. X. et al. Nitrogen-fixing and plant growth-promoting ability of two endophytic bacterial strains isolated from sugarcane stalks. Sugar Tech, India, v. 18, n. 4, p. 373-379, 2016.

Yoolong, S. et al. Modulation of salt tolerance in Thai jasmine rice (Oryza sativa L. cv. KDML105) by Streptomyces venezuelae ATCC 10712 expressing ACC deaminase. Scientific Reports, London, v. 9, n. 1, 2019.

Zaheer, M. S. et al. Investigating the effect of Azospirillum brasilense and Rhizobium pisi on agronomic traits of wheat (Triticum aestivum L.). Archives of Agronomy and Soil Science, Abingdon, Oxfordshire, v. 65 , n. 11, p. 1-11, 2019.

Zarei, T. et al. Improving sweet corn (Zea mays L. var saccharata) growth and yield using Pseudomonas fluorescens inoculation under varied watering regimes. Agricultural Water Management, Amsterdam, v. 226, p. 105757, 2019.

Zhang, G. et al. Effects of the inoculations using bacteria producing ACC deaminase on ethylene metabolism and growth of wheat grown under different soil water contents. Plant Physiology and Biochemistry, Netherlands, v. 125, p. 178-184, 2018.

Recebido: 06 ago. 2020 Aprovado: 27 out. 2020 